\title{
Delocalization effect of the Hubbard repulsion in exact terms and two dimensions
}

\author{
Zsolt Gulácsi \\ Department of Theoretical Physics, University of Debrecen, H-4010 Debrecen, Hungary \\ (Received 26 February 2008; revised manuscript received 21 April 2008; published 11 June 2008)
}

\begin{abstract}
The genuine physical reasons explaining the delocalization effect of the Hubbard repulsion $U$ are analyzed. First, it is shown that always when this effect is observed, $U$ acts on the background of a macroscopic degeneracy present in a multiband type of system. After this step, I demonstrate that acting in such conditions, by strongly diminishing the double occupancy, $U$ spreads out the contributions in the ground state wave function, hence strongly increases the one-particle localization length, and consequently extends the oneparticle behavior producing conditions for a delocalization effect. To be valuable, the reported results are presented in exact terms, being based on the first exact ground states deduced at half-filling in two dimensions for a prototype two band system, which is the generic periodic Anderson model at finite value of the interaction.
\end{abstract}

DOI: 10.1103/PhysRevB.77.245113

PACS number(s): 71.10.Fd, 71.27.+a, 71.10.Hf

\section{INTRODUCTION}

The ability of the Hubbard repulsion $U$ to drive transitions to insulating behavior is widely studied and known. ${ }^{1}$ However, in the recent years, considerable interest is devoted to the study of the converse phenomenon, namely, the delocalization effect produced by $U .^{2-4}$ This effect attracts much attention given by the unexpected nature of the process, since it can finally lead to an interaction driven metallic behavior starting from an insulator, instead to drive transitions to insulating behavior from a metal, as intuitively is expected. The interest for understanding the delocalization effect of $U$ has been first set up by the observed conducting behavior in two-dimensional (2D) disordered systems ${ }^{5}$ where the electrons are localized in the starting noninteracting phase by disorder. However, as opposed to the random case, periodic external potential is also able to cause insulating behavior, hence can be as well used as a starting point in investigating the delocalization effect of $U$. On this line, the ionic Hubbard model is investigated at half-filling in different approximations, or numerical methods, starting from dynamical mean field theory, ${ }^{2}$ its cluster version, ${ }^{4}$ or quantum Monte Carlo technique, ${ }^{3}$ obtaining insulator to metal transition in $2 \mathrm{D}$, even if several aspects of the results are under debate. $^{4}$

On the other side, the clear physical reason for the $U$ produced delocalization is far to be properly understood. Up to this moment, only correlation screening of the one-body potential responsible for the insulating phase ${ }^{2}$ has been invoked as an intuitive possibility for explanation.

In order to understand the physical reasons for a possible insulator to metal transition caused by the Hubbard $U$, we have to pay attention to two aspects that must concur in producing a conducting behavior starting from an insulator: ${ }^{6}$ (i) the one-particle states must become extended and (ii) the gap in the charge excitation spectrum must disappear. The correlation screening of the one-body potential can be, in principle, a physical insight in explaining point (ii), but it does not explain how, given by $U$, point (i) becomes also satisfied, e.g., how the state becomes extended given by the Hubbard interaction in a genuine multielectronic case. The deep physical reasons of this last process are not clearly known, and in my knowledge have not been directly investigated so far at a genuine multielectronic level in conditions of interest here, e.g., around half-filling.

The purpose of the presented paper is to provide valuable information regarding this issue. In this paper, we aim to answer the question is there a direct evidence that $U$, in the studied circumstances, is able to extend one-particle states. To obtain a valuable answer, the description is made here at exact level in 2D. Our strategy is to deduce in a nonapproximated manner the exact ground states in conditions of interest. Such obtained state will be a genuine multielectronic state, hence usually, is not possible to rigorously discuss about one-particle states in its frame. However, based on it, one-particle behavior can be tested by calculating the expectation value of the long-range hopping type of correlations,

$$
\Gamma(\mathbf{r})=\frac{1}{N_{\Lambda}} \sum_{\mathbf{i}=1}^{N_{\Lambda}} \Gamma_{\mathbf{i}}(\mathbf{r}), \quad \Gamma_{\mathbf{i}}(\mathbf{r})=(1 / 2) \sum_{\sigma} \mid\left\langle\hat{b}_{\mathbf{i}, \sigma}^{\dagger} \hat{b}_{\mathbf{i}+\mathbf{r}, \sigma}+\text { H.c. }\right\rangle \mid,
$$

where $N_{\Lambda}$ represents the number of lattice sites and $\hat{b}_{\mathbf{i}, \sigma}^{\dagger}$ creates an electron at site $\mathbf{i}$ with spin $\sigma$. The study will be given in this paper for the nondisordered case in homogeneous conditions, hence $\Gamma(\mathbf{r})=\Gamma_{\mathrm{i}}(\mathbf{r})$ holds. During such a study, the one-particle localization length $\ell$ can also be introduced by $\Gamma(\mathbf{r}) \sim \exp (-|\mathbf{r}| / \ell)$. In order to answer the question aimed to be investigated here, one simply must analyze whether, or not, $U$ is able to increase $\Gamma(\mathbf{r})$ and $\ell$ in the conditions of interest.

In order to fix the frame of the study, we must clarify the nature of the circumstances of interest. On this line, one observes that in both cases mentioned in the introductory part, the $U$ driven delocalization effect emerges on the background of a macroscopic degeneracy. Indeed, in the disordered cases, the ground states are macroscopically degenerate, ${ }^{7}$ while in the ionic Hubbard model case, the effects are observed in the neighborhood of the point where the single occupancy at one-one site of sublattices $A$ and $B$ has the same energy as an empty site in $A$ and a double occupied 
site in $B .{ }^{3}$ Furthermore, one knows that the ionic Hubbard model is of two band type, ${ }^{8}$ while real materials, also disordered once, have a multiband structure which one usually projects in a few band picture. ${ }^{9}$ Here, this projection is stopped for convenience at the two band level.

Given by these observations, one realizes that in order to find the physical insights of interest here, one must consider a prototype two band model in $2 \mathrm{D}$. The noninteracting case must be set in the position of a macroscopic degeneracy from the point of view of the one-particle states such to find an insulator at $U=0$. This can be achieved by choosing the Hamiltonian parameters to provide a bare flat (e.g., macroscopically degenerate) lowest band, which is separated by a finite gap from the upper dispersive bare band, and fixing the filling of the system to half (e.g., lowest band: completely filled; upper band separated by a gap: empty). After this step, one turns on $U$ and analyzes its effect.

This experience is quite interesting also because one knows that turning on the interaction in many-body systems with macroscopic degeneracy, one always finds a fascinating physics as, for example, in the case of the Laughlin state in two dimensions, ${ }^{10}$ Hubbard chains with bare degenerate bands, ${ }^{11}$ or geometrically frustrated spin systems. ${ }^{12}$

The prototype two band model chosen for the study in this paper will be the periodic Anderson model (PAM), simply because several technical aspects regarding its ground state construction are known. ${ }^{13,14}$ The PAM has been constructed to be the simplest microscopic model used to investigate the characteristic properties of heavy fermion and intermediatevalence compounds containing elements with incompletely filled $f$ shells ${ }^{13,14}$ and serves as well, as a test prototype two band model used in various circumstances of interest. ${ }^{7,15,16}$ The PAM consists of strongly correlated, almost localized $f$ electrons that experience an on-site Coulomb repulsion $U$ $>0$ and hybridize with a band of noninteracting conduction $(d-)$ electrons. In contrast to the one-dimensional Hubbard model, PAM is not integrable even in dimension $D=1$. Therefore, our knowledge of the physics described by the PAM at finite and nonzero $U$ is almost exclusively obtained by approximate methods. Notable exceptions are the constructed exact ground states in dimensions $D=1,2,3$ at and below $1 / 4$ or at and above $3 / 4$ filling, ${ }^{13,14,17-19}$ e.g., $\bar{n}$ $=N /\left(4 N_{\Lambda}\right) \in(0,1 / 4] \cup[3 / 4,1)$, where $N$ is the number of electrons. The physically quite interesting region situated at and in the vicinity of half-filling, which is strongly debated at the level of different approximations or numerical methods, ${ }^{20-22}$ lacks exact results for $0<U<\infty$.

The used technique casts the Hamiltonian in a positive semidefinite form, ${ }^{13}$ and the fact that a such expression has the possible minimum eigenvalue zero represents the route in obtaining the exact ground states. ${ }^{23}$ The method can be applied even in unexpected situations in the context of exact solutions, as ${ }^{14} D=3$, disordered and interacting systems in $2 \mathrm{D},{ }^{7}$ nonintegrable Hubbard chains in external fields, ${ }^{11}$ or stripe, checkerboard and droplet ground states ${ }^{15}$ in 2D. However, given by strong correlation effects not treatable mathematically up to this moment in the studied concentration region, it has not yet been applied for the PAM at finite $U$ $\neq 0$ around half-filling.

The deduced results provide the first exact ground states for the 2D generic PAM at half-filling and finite value of the interaction. This is achieved by the use of extended operators in constructing the ground state, a technique which is also described in the context of nonintegrable systems in this paper.

The comparison of ground states at $U=0$ and $U>0$ on the line of Eq. (1) shows that the Hubbard repulsion, by strongly diminishing the double occupancy, spreads out the contributions in the ground state, hence is able to increase by several order of magnitudes the one-particle localization length. Hence, the one-particle behavior in the analyzed conditions is extended by $U$, which is a clear physical source of a delocalization effect. Consequently, the answer to the question we are looking for in this paper is "yes." I also note that one already knows that even away from half-filling, $U$ will be able to create in relatively similar conditions subtle correlated conducting phases. ${ }^{11,13,14}$ It is also known that insulator to metal transition can be driven as well by the disorder, ${ }^{24}$ with the process emerging at half-filling again from a strongly degenerate insulating phase.

The remaining part of the paper is structured as follows. Section II, describes the model and its solution in the noninteracting case. Section III prepares the deduction by transforming the starting Hamiltonian in a positive semidefinite form. Section IV describes the extended operators that allow the construction of the exact ground states. Section V presents the deduced exact ground state, which characterizes as well the physical properties of the system, while Sec. VI contains the summary and conclusions that close the presentation.

\section{MODEL USED}

\section{A. Hamiltonian}

I analyze a generic PAM Hamiltonian $\hat{H}=\hat{H}_{0}+U \hat{U}_{f}$ defined on an $N_{\Lambda}=L \times L$, with $2 \mathrm{D}$ Bravais lattice with primitive vectors $(\mathbf{x}, \mathbf{y})$. The Hubbard term is $\hat{U}_{f}=\sum_{\mathbf{i}} \hat{n}_{\mathbf{i}, \uparrow}^{f} \hat{n}_{\mathbf{i}, \downarrow}^{f}$, arbitrary $U>0$ is considered, and

$$
\begin{aligned}
\hat{H}_{0}= & \sum_{\mathbf{i}, \sigma}\left\{\left(\sum_{\mathbf{r}_{d}} t_{\mathbf{r}_{d}} \hat{d}_{\mathbf{i}, \sigma}^{\dagger} \hat{d}_{\mathbf{i}+\mathbf{r}_{d}, \sigma}+V_{0} \hat{d}_{\mathbf{i}, \sigma}^{\dagger} \hat{f}_{\mathbf{i}, \sigma}\right.\right. \\
& \left.\left.+\sum_{\mathbf{r}=\mathbf{x}, \mathbf{y}} \sum_{b, b^{\prime}=d, f ; b \neq b^{\prime}} V_{\mathbf{r}}^{b, b^{\prime}} \hat{b}_{\mathbf{i}, \sigma}^{\dagger} \hat{b}_{\mathbf{i}+\mathbf{r}, \sigma}^{\prime}+\text { H.c. }\right)+E_{f} \hat{f}_{\mathbf{i}, \sigma}^{\dagger} \hat{f}_{\mathbf{i}, \sigma}\right\},
\end{aligned}
$$

where in order to describe real systems, the hopping matrix element $t_{\mathbf{r}_{d}}$ covers by the index $\mathbf{r}_{d}$ nearest and next nearest neighbors, $V_{0}$ and $V_{\mathbf{r}}^{b, b^{\prime}}, b, b^{\prime}=d, f$, give the strengths of the on-site and nearest neighbor hybridizations, and $E_{f}$ represents the on-site $f$-electron energy. During calculations, periodic boundary conditions are used, and the filling is fixed to half (e.g., $\bar{n}=1 / 2$ and $N=2 N_{\Lambda}$ ).

\section{B. $U=0$ noninteracting case}

In order to have a macroscopic degeneracy at one-particle level, one considers first the noninteracting $U=0$ case and selects the $\hat{H}_{0}$ parameters such to obtain a lowest flat band in the noninteracting band structure. For simplicity, one consid- 
ers the $(x, y)$ symmetric case $t_{1}=t_{\mathbf{x}}=t_{\mathbf{y}}, t_{2} / 2=t_{2 \mathbf{x}}=t_{2 \mathbf{y}}$ $=t_{\mathbf{y} \pm \mathbf{x}} / 2$, and $V_{1}=V_{\mathbf{x}}^{b, b^{\prime}}=V_{\mathbf{y}}^{b, b^{\prime}}$. To have a lowest flat band in the noninteracting band structure, one takes

$$
V_{1} t_{1}=V_{0} t_{2}, \quad E_{f}=2 \frac{V_{1}^{2}}{t_{2}}-\frac{V_{0}^{2} t_{2}}{2 V_{1}^{2}}-2 t_{2}, \quad t_{2}>0 .
$$

Indeed, for condition (3), $\hat{H}_{0}$ from Eq. (2) transformed in $\mathbf{k}$ space can be diagonalized into the form

$$
\hat{H}_{0}=\sum_{\sigma} \sum_{\mathbf{k}}\left[E_{1, \mathbf{k}} \hat{C}_{1, \mathbf{k}, \sigma}^{\dagger} \hat{C}_{1, \mathbf{k}, \sigma}+E_{2, \mathbf{k}} \hat{C}_{2, \mathbf{k}, \sigma}^{\dagger} \hat{C}_{2, \mathbf{k}, \sigma}\right],
$$

where $\hat{C}_{\nu, \mathbf{k}, \sigma}^{\dagger}$ represents the canonical Fermi operator of the $\nu$ th diagonalized band (here, $\nu=1,2)$ of $\hat{H}_{0}$. In the case of Eq. (3), the two bands, $E_{1, \mathbf{k}}=-\left(t_{2} / 2\right)\left[\left(V_{0}^{2} / V_{1}^{2}\right)+4\right]$ $<E_{2, \mathbf{k}}=E_{1, \mathbf{k}}+2 t_{2}\left\{\left(V_{1} / t_{2}\right)^{2}+\left[C_{\mathbf{k}}+V_{0} /\left(2 V_{1}\right)\right]^{2}\right\}, \quad C_{\mathbf{k}}=\cos (\mathbf{x k})$ $+\cos (\mathbf{y k})$, are clearly separated, the lowest band is flat, and the band gap being $\Delta=2 t_{2}\left[\left(V_{0} / t_{1}\right)^{2}+\left(\left|V_{0} / 2 V_{1}\right|-2\right)^{2}\right] \neq 0$. Consequently, in the noninteracting case, given by the presence of the lowest bare flat band, a macroscopic degeneracy is present, whose degree at the one-particle level is $2 N_{\Lambda}$.

The canonical Fermi operators present in Eq. (4) are given by

$$
\begin{aligned}
& \hat{C}_{1, \mathbf{k}, \sigma}^{\dagger}=\frac{1}{\sqrt{2}}\left[\sqrt{1+F_{\mathbf{k}}} \hat{d}_{\mathbf{k}, \sigma}^{\dagger}-\operatorname{sgn}\left(\alpha_{\mathbf{k}}\right) \sqrt{1-F_{\mathbf{k}}} \hat{f}_{\mathbf{k}, \sigma}^{\dagger}\right], \\
& \hat{C}_{2, \mathbf{k}, \sigma}^{\dagger}=\frac{1}{\sqrt{2}}\left[\sqrt{1-F_{\mathbf{k}}} \hat{d}_{\mathbf{k}, \sigma}^{\dagger}+\operatorname{sgn}\left(\alpha_{\mathbf{k}}\right) \sqrt{1+F_{\mathbf{k}}} \hat{f}_{\mathbf{k}, \sigma}^{\dagger}\right],
\end{aligned}
$$

where $\alpha_{\mathbf{k}}=V_{0}+2 V_{1} C_{\mathbf{k}}, \beta_{\mathbf{k}}=V_{1}^{2} / t_{2}-t_{1}^{2} /\left(4 t_{2}\right)-t_{1} C_{\mathbf{k}}-t_{2} C_{\mathbf{k}}^{2}$, and $F_{\mathbf{k}}=\left[1+\left(\alpha_{\mathbf{k}} / \beta_{\mathbf{k}}\right)^{2}\right]^{-0.5}$. The ground state at $U=0$ and halffilling (lowest band: completely filled; upper band: completely empty) becomes

$$
\left|\Psi_{g}^{0}\right\rangle=\prod_{\sigma} \prod_{\mathbf{k}=1}^{N_{\Lambda}} \hat{C}_{1, \mathbf{k}, \sigma}^{\dagger}|0\rangle,
$$

which represents a paramagnetic band insulator. The ground state energy corresponding to Eq. (6) is $E_{g}^{0}\left(2 L^{2}\right)=$ $-t_{2} L^{2}\left[\left(V_{0}^{2} / V_{1}^{2}\right)+4\right]$.

Denoting by $\langle\cdots\rangle_{0}$, which is ground state expectation values calculated in terms of Eq. (6), for each site $\mathbf{i}$ of the system, one finds $d_{\mathbf{i}}^{f}=\left\langle\hat{n}_{\mathbf{i}, \uparrow}^{f} \hat{n}_{\mathbf{i}, \downarrow}^{f}\right\rangle_{0} \neq 0$. Concerning $\Gamma(\mathbf{r})$ from Eq. (1), using Eq. (6), $b=d, f$, one finds $\Gamma(\mathbf{r}) \sim \exp (-|\mathbf{r}| / \ell)$, where the associated one-particle localization length $\ell$ is of order unity in lattice constant units (for example, for $\mathbf{r} \| \mathbf{x}$, one obtains $\ell /|\mathbf{x}|=0.5$ for the Hamiltonian parameters fixed in Fig. 8). Based on this result, one concludes that the noninteracting system in the studied conditions represents a localized band insulator.

\section{HAMILTONIAN TRANSFORMED IN A POSITIVE SEMIDEFINITE FORM}

\section{A. Transformed Hamiltonian}

In order to deduce the exact ground state in the interacting case, one first transforms the Hamiltonian $\hat{H}=\hat{H}_{0}+U \hat{U}_{f}, U$

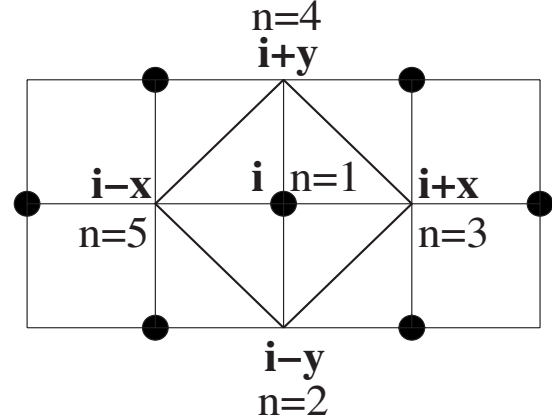

FIG. 1. The cell defined at site $\mathbf{i}$ (thick line) containing five sites of the lattice $(\mathbf{i}, \mathbf{i} \pm \mathbf{x}, \mathbf{i} \pm \mathbf{y})$ on which the operator $\hat{A}_{\mathbf{i}, v, e}$ is defined. The sites of the sublattice $v, \mathbf{i} \in S_{v}$, are denoted by black dots, $(\mathbf{x}, \mathbf{y})$ are the primitive vectors of the lattice, and $n=1,2, \ldots, 5$ represents the notation of sites inside the cell.

$>0$, given in Eqs. (2) and (3) in a positive semidefinite form. I note that this exact transformation is $N$ independent and provides

$$
\begin{gathered}
\hat{H}=\hat{H}_{0}+U \hat{U}_{f}=\hat{G}+U \hat{U}_{f}+E_{g}(N), \\
\hat{G}=\sum_{v=1,2} \sum_{e= \pm 1} \sum_{\mathbf{i} \in S_{v}} \hat{A}_{\mathbf{i}, v, e}^{\dagger} \hat{A}_{\mathbf{i}, v, e}, \quad E_{g}(N)=-K_{d} N .
\end{gathered}
$$

To clarify Eq. (7), the following explications must be given: In the first line of Eq. (7), $\hat{G}$ and $U \hat{U}_{f}$ are positive semidefinite operators, while $E_{g}(N)$ represents the ground state energy (see Sec. IV), with a number that has a given dependence on the Hamiltonian parameters and whose expression will be clarified below. In order to obtain Eq. (7), first we divide the lattice into two sublattices. The sublattices are denoted by $v=1,2$, and all lattice sites from the sublattice $v$ are denoted by $S_{v}$. After this step, one introduces the cell operators $\hat{A}_{\mathbf{i}, v, e}$, which are given by

$$
\hat{A}_{\mathbf{i}, v, e}=a_{1, f} \hat{F}_{\mathbf{i}, \lambda_{v}, e}+\sum_{n=1}^{5} a_{n, d} \hat{D}_{\mathbf{i}+\mathbf{r}_{n}, \lambda_{v}, e} .
$$

Even if labeled by the site $\mathbf{i}$, the $\hat{A}_{\mathbf{i}, v, e}$ operators are not local and collect contributions in the form of a linear combination from five sites situated around the site i. These five sites denoted by $\mathbf{i}+\mathbf{r}_{n}$, where $n=1,2,3,4,5$, build up together the cell defined at the site $\mathbf{i}$, where taken for increasing $n, \mathbf{r}_{n}$ has the values $0,-\mathbf{y}, \mathbf{x}, \mathbf{y}$, and $-\mathbf{x}$ (see Fig. 1). After this step, one presents below separately in detail the numerical prefactors and the operators present in Eq. (8).

\section{B. Numerical prefactors of $\hat{A}_{\mathrm{i}, v, e}$}

The numerical coefficients $a_{1, f}, a_{n, d}$ in Eq. (8) are related to the Hamiltonian parameters of $\hat{H}_{0}$, being given by the following system of equations:

$$
\begin{gathered}
t_{\mathbf{x}}=a_{1, d}^{*} a_{3, d}+a_{5, d}^{*} a_{1, d}, \quad t_{\mathbf{y}}=a_{1, d}^{*} a_{4, d}+a_{2, d}^{*} a_{1, d}, \\
t_{\mathbf{y} \pm \mathbf{x}}=a_{2, d}^{*} a_{4 \mp 1, d}+a_{4 \pm 1, d}^{*} a_{4, d}, \quad V_{0}=a_{1, d}^{*} a_{1, f},
\end{gathered}
$$




$$
\begin{gathered}
t_{2 \mathbf{x}}=a_{5, d}^{*} a_{3, d}, \quad t_{2 \mathbf{y}}=a_{2, d}^{*} a_{4, d}, \quad V_{\mathbf{x}}^{d f}=a_{5, d}^{*} a_{1, f}, \\
V_{\mathbf{y}}^{d f}=a_{2, d}^{*} a_{1, f}, \quad V_{\mathbf{x}}^{f d}=a_{1, f}^{*} a_{3, d}, \quad V_{\mathbf{y}}^{f d}=a_{1, f}^{*} a_{4, d}, \\
E_{f}=\left|a_{1, f}\right|^{2}-K_{d}, \quad K_{d}=\sum_{n=1}^{5}\left|a_{n, d}\right|^{2} .
\end{gathered}
$$

It is important to note that the exact transformation presented in Eq. (7) works only if the "matching conditions" [Eq. (9)] are satisfied. I also mention that the system of Eq. (9), through the expression of $K_{d}$, fixes as well the value of the ground state energy $E_{g}(N)$ in Eq. (7). One obtains the system of Eq. (9) by: (1) calculating $\hat{A}_{\mathbf{i}, v, e}^{\dagger} \hat{A}_{\mathbf{i}, v, e}$ based on Eqs. (8) and (11), (2) deducing $\hat{G}+E_{g}(N)$ in Eq. (7) starting from the previous result, and (3) finally, equating to $\hat{H}_{0}$ from Eq. (2) the obtained expression. I note that the presence of $\hat{f}_{\mathbf{j}, \sigma}$ operator only in the middle of the cell operator $\hat{A}_{\mathbf{i}, v, e}$ in Eq. (8) (see also Fig. 1) leads to the absence of the direct $f$-electron hopping in the transformed Hamiltonian.

The matching conditions [Eq. (9)], in the $(x, y)$ symmetric case specified above Eq. (3), give solution only when Eq. (3) is satisfied. The solution is characterized by $a_{n \geq 2, d}=a_{2, d}$, and one obtains

$$
a_{1, f}=V_{1} \frac{\sqrt{2}}{\sqrt{t_{2}}} e^{i \phi}, \quad a_{1, d}=\frac{V_{0}}{V_{1}} \frac{\sqrt{t_{2}}}{\sqrt{2}} e^{i \phi}, \quad a_{2, d}=\frac{\sqrt{t_{2}}}{\sqrt{2}} e^{i \phi},
$$

where $\phi$ is an arbitrary phase.

\section{Operators contained in $\hat{A}_{\mathrm{i}, v, e}$}

The operators $\hat{D}_{\mathbf{j}, \lambda_{v}, e}$ and $\hat{F}_{\mathbf{j}, \lambda_{v}, e}$, where $e= \pm 1$, are genuine canonical Fermi operators substituting the starting canonical Fermi operators $\hat{d}_{\mathbf{j}, \sigma}$ and $\hat{f}_{\mathbf{j}, \sigma}$, where $\sigma=\uparrow, \downarrow$. Their expression is given by the following relations:

$$
\begin{aligned}
& \hat{B}_{\mathbf{j}, \lambda_{v},+1}= \frac{\left|\lambda_{v}\right|}{\sqrt{1+\left|\lambda_{v}\right|^{2}}}\left(\hat{b}_{\mathbf{j}, \uparrow}+\frac{1}{\lambda_{v}} \hat{b}_{\mathbf{j}, \downarrow}\right), \\
& \hat{B}_{\mathbf{j}, \lambda_{v},-1}=\frac{\left|\lambda_{v}\right|}{\sqrt{1+\left|\lambda_{v}\right|^{2}}}\left(\frac{1}{\lambda_{v}^{*}} \hat{b}_{\mathbf{j}, \uparrow}-\hat{b}_{\mathbf{j}, \downarrow}\right),
\end{aligned}
$$

where for $b=d(f)$, one has $B=D(F)$ and $\lambda_{v}$ is an arbitrary complex number.

Looking for the physical meaning of $\hat{B}$ operators, taking, for example, the $b=d$ case, using the fermionic spin representation for $1 / 2$ spin operators, $\hat{S}_{d, \mathbf{i}}^{z}=(1 / 2)\left(\hat{n}_{\mathbf{i}, \uparrow}^{d}\right.$ $\left.-\hat{n}_{\mathbf{i}, \downarrow}^{d}\right), \hat{S}_{d, \mathbf{i}}^{+}=\hat{d}_{\mathbf{i}, \uparrow}^{\dagger} \hat{d}_{\mathbf{i}, \downarrow}, \hat{S}_{d, \mathbf{i}}^{-}=\hat{d}_{\mathbf{i}, \downarrow}^{\dagger} \hat{d}_{\mathbf{i}, \uparrow}, \hat{S}_{d, \mathbf{i}}^{ \pm}=\hat{S}_{d, \mathbf{i}}^{x} \pm i \hat{S}_{d, \mathbf{i}}^{y}, \quad \hat{S}_{d, \mathbf{i}}^{2}=\hat{S}_{d, \mathbf{i}}^{z} \hat{S}_{d, \mathbf{i}}^{z}$ $+(1 / 2)\left(\hat{S}_{d, \mathbf{i}}^{+} \hat{S}_{d, \mathbf{i}}^{-}+\hat{S}_{d, \mathbf{i}}^{-} \hat{S}_{d, \mathbf{i}}^{+}\right)$, and introducing the states, $\left|D_{\mathbf{i}, \lambda, e}\right\rangle$ $=\hat{D}_{\mathbf{i}, \lambda, e}^{\dagger}|0\rangle,\left\langle D_{\mathbf{i}, \lambda, e} \mid D_{\mathbf{i}, \lambda, e}\right\rangle=1$, one finds for arbitrary $\lambda$

$$
\hat{S}_{d, \mathbf{i}}^{2}\left|D_{\mathbf{i}, \lambda, e}\right\rangle=\frac{3}{4}\left|D_{\mathbf{i}, \lambda, e}\right\rangle, \quad\left(\hat{n}_{\mathbf{i}, \uparrow}^{d}+\hat{n}_{\mathbf{i}, \downarrow}^{d}\right)\left|D_{\mathbf{i}, \lambda, e}\right\rangle=\left|D_{\mathbf{i}, \lambda, e}\right\rangle .
$$

Since similar relations can also be written for the $b=f$ case, one concludes that $\hat{B}_{\mathbf{i}, \lambda, e}^{\dagger}$ introduces one spin $1 / 2$ fermion $(b$ $=d$ or $b=f$ for $B=D$ or $B=F$ ) on the site i. One further observes that at the level of expectation values, the spin direction and orientation are provided by $\lambda$ and $e$. Indeed, for all $\alpha=x, y, z$, one has $\left\langle D_{\mathbf{i}, \lambda,-1}\left|\hat{S}_{d, \mathbf{i}}^{\alpha}\right| D_{\mathbf{i}, \lambda,-1}\right\rangle=-\left\langle D_{\mathbf{i}, \lambda, 1}\left|\hat{S}_{d, \mathbf{i}}^{\alpha}\right| D_{\mathbf{i}, \lambda, 1}\right\rangle$, and

$$
\begin{aligned}
\left\langle D_{\mathbf{i}, \lambda, 1}\left|\hat{S}_{d, \mathbf{i}}^{\alpha}\right| D_{\mathbf{i}, \lambda, 1}\right\rangle= & \frac{1}{1+|\lambda|^{2}}\left[\delta_{x, \alpha} \operatorname{Re}(\lambda)+\delta_{y, \alpha} \operatorname{Im}(\lambda)\right. \\
& \left.+\delta_{z, \alpha} \frac{|\lambda|^{2}-1}{2}\right]
\end{aligned}
$$

such that $\left(\left\langle D_{\mathbf{i}, \lambda, e}\left|\vec{S}_{d, \mathbf{i}}\right| D_{\mathbf{i}, \lambda, e}\right\rangle\right)^{2}=1 / 4$. These results show that, in fact, $\lambda$ fixes an arbitrary axis along which the spin is placed, while $e= \pm 1$ gives the spin direction along the fixed axis. Indeed, for example, at $\lambda=1$, one has $\hat{S}_{d, \mathbf{i}}^{x}\left|D_{\mathbf{i}, \lambda=1, e}\right\rangle$ $=(e / 2)\left|D_{\mathbf{i}, \lambda=1, e}\right\rangle, \quad$ for $\quad \lambda=i, \quad$ one obtains $\hat{S}_{d, \mathbf{i}}^{y}\left|D_{\mathbf{i}, \lambda=i, e}\right\rangle$ $=(e / 2)\left|D_{\mathbf{i}, \lambda=i, e}\right\rangle$, while $\lambda \rightarrow \infty$ corresponds to the $\hat{S}_{d, \mathbf{i}}^{z}\left|D_{\mathbf{i}, \lambda, e}\right\rangle$ $=(e / 2)\left|D_{\mathbf{i}, \lambda, e}\right\rangle$ case (e.g., for $\lambda=1, \lambda=i$, and $\lambda \rightarrow \infty$, the spin axis becomes in order the $x, y$, and $z$ axes).

I further note that the relation $\hat{D}_{\mathbf{i}, \lambda, 1}^{\dagger} \hat{D}_{\mathbf{i}, \lambda,-1}^{\dagger}=\hat{d}_{\mathbf{i}, \downarrow}^{\dagger} \hat{d}_{\mathbf{i}, \uparrow}^{\dagger}$ holds for all $\lambda$. It is also important to mention that in Eq. (7), after the transformation of the starting Hamiltonian defined by Eq. (2), for $v=1,2$, one obtains arbitrary but fixed $\lambda_{1}$ and $\lambda_{2}$.

\section{DESCRIPTION OF THE MATHEMATICAL SOLUTION THAT LEADS TO THE GROUND STATE WAVE FUNCTION AT HALF-FILLING AND $U>0$}

In Eq. (7), one has the Hamiltonian transformed in a positive semidefinite form which now must be analyzed in order

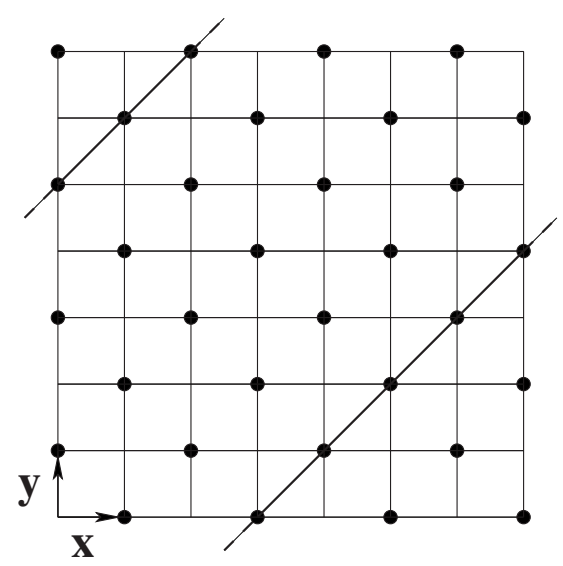

FIG. 2 . An $8 \times 8$ lattice (e.g., $L=8$ holds) containing one straight line $l_{\mathbf{y}+\mathbf{x}, v=1, n}$ (thick line). The direction of the line is $\mathbf{x}+\mathbf{y}$, it is contained in the sublattice $v=1$ denoted by full circles, the line contains $L=8$ sites, and note that periodic boundary conditions are considered. $(\mathbf{x}, \mathbf{y})$ are the primitive vectors of the $2 \mathrm{D}$ Bravais lattice. In the $\mathbf{x}+\mathbf{y}$ direction $L / 2=4$, such lines can be plotted. 


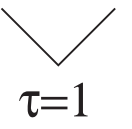

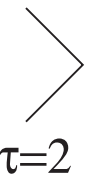

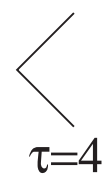

FIG. 3. The four possible breaking directions denoted by the index $\tau$.

to deduce its ground state at half-filling in the interacting $U>0$ case. As mentioned above [Eq. (2)], $\hat{H}$ has been defined on an $L \times L$ Bravais lattice, and obtaining the transformed form in Eq. (7), the lattice has been divided into two sublattices $v=1,2$ containing each $L^{2} / 2$ sites.

In deducing the ground state at $N$ particles, we have to find a wave vector $|\phi(N)\rangle=\hat{K}^{\dagger}|0\rangle$, where $\hat{K}^{\dagger}$ introduces $N$ electrons into the system, and based on Eq. (7),

$$
\hat{G}|\phi(N)\rangle=\hat{U}_{f}|\phi(N)\rangle=0
$$

holds. In such conditions, $|\phi(N)\rangle$ represents the ground state of the $N$-electron system described by $\hat{H}$ in Eq. (7), with the ground state energy being $E_{g}(N)$. In order to prove Eq. (14), one shows $\left\{\hat{A}_{\mathbf{i}, v, e}, \hat{K}^{\dagger}\right\}=0$ for the first part, and demonstrate that $|\phi(N)\rangle$ does not contain double $f$ occupancy for the second part.

Below, I describe the $\hat{K}^{\dagger}$ operator that will provide the ground state at $N=2 L^{2}$ (half-filling). Since in this concentration region strong correlation effects are present, $\hat{K}^{\dagger}$ has a relatively complicated form. Because of this fact, in describing $\hat{K}^{\dagger}$, first I present it, describe it in details, give its expression, and explain how it was deduced.

\section{A. Mathematical key of the solution}

Taking $|0\rangle$ as the bare vacuum, one considers the state

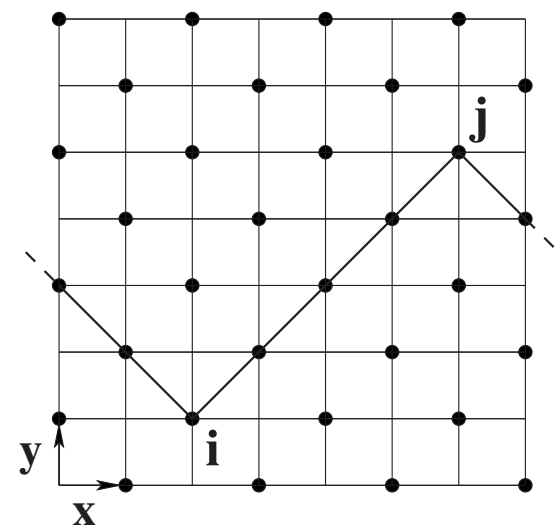

FIG. 4. A broken line $l_{v}\left(\mathbf{i}, \tau_{\mathbf{i}}=1\right)$ (thick line) defined at the site $\mathbf{i}$ in a $8 \times 8$ lattice. $l_{v}\left(\mathbf{i}, \tau_{\mathbf{i}}=1\right)$ connects sites of the $v$ sublattice (full dots). Note that a number of $L^{2} / 2$ such broken lines can be plotted for a fixed $v$.

$$
\left|\Psi_{e_{1}, e_{2}}^{\lambda_{1}, \lambda_{2}}\right\rangle=\prod_{v=1}^{2} \prod_{\mathbf{i} \in S_{v}}\left(\hat{W}_{v, \mathbf{i}, \tau=1, \lambda_{v}, e_{v}}^{\dagger} \hat{W}_{v, \mathbf{i}, \tau=4, \lambda_{v}, e_{v}}^{\dagger}\right)|0\rangle,
$$

where $\hat{W}_{v, \mathbf{i}, \tau, \lambda_{v}, e_{v}}^{\dagger}$ is the broken line operator defined for the sublattice $v$, labeled by the site $\mathbf{i}$ of $S_{v}$, breaking direction at the site $\mathbf{i}\left(\tau_{\mathbf{i}}=\tau\right)$, spin direction given by $\lambda_{v}$, and spin orientation specified by $e_{v}$. One arrives to the broken line operator notion by looking for the desired $2 L^{2}$ number of linearly independent operators suitable in constructing the ground state at $\bar{n}=1 / 2$. If we would like to use an operator of the type $\hat{C}_{1, \mathbf{k}, \sigma}^{\dagger}$ (or its Fourier transform in $\mathbf{r}$ space) from Eqs. (5) and (6) for this job, we shortly realize that only $L^{2}$ operators are available in constructing the ground state at $U>0$, namely, those with fixed $\sigma$, since mixing the spin index, automatically double occupancy occurs which raises the energy. Hence, the expression of the interacting ground state requires new operators $\hat{W}^{\dagger}$ which must be find. The search for these leads first to operators constructed along lines in the $\mathbf{y}+\mathbf{x}$ direction. These resemble to the operators presented in Fig. 7, but their number is of order $L$, hence is small. At this point, one remembers that by breaking a straight line, a site (e.g., the breaking point) becomes attached to the line, the number of sites being $L^{2}$. Hence, in this manner, it is possible to reach the number of desired operators. The presence of the cusp point on the line complicates a little the expression of the operators, but this is, in fact, the way one reaches the broken line operator notion. Since the structure of the $W^{\dagger}$ operator is not transparent in Eq. (15), I explain it in detail below.

\section{B. Straight lines and broken lines}

Connecting the sites of a given sublattice in the $\mathbf{y}+\mathbf{x}$ direction, $n \in[1, L / 2]$, different straight lines $l_{\mathbf{y}+\mathbf{x}, v, n}$ can be drawn, each containing $L$ sites (see Fig. 2). For simplicity, one considers below even $L$.

Now, one breaks the straight lines at an arbitrary site $\mathbf{i} \in l_{\mathbf{y}+\mathbf{x}, v, n}$. In order this to be possible, one first defines four breaking directions denoted by the index $\tau$, as shown in Fig. 3 . If one fixes the breaking direction at $\mathbf{i}$ by $\tau_{\mathbf{i}}=1$, one finds the "broken line" as presented in Fig. 4.

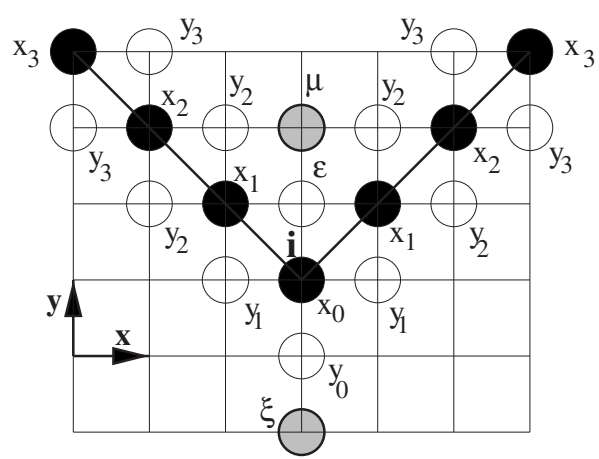

FIG. 5. The first cusp region of $\hat{W}_{v=1, \mathbf{i}, \tau=1, \lambda_{v}, e_{v}}^{\dagger}$ constructed along the broken line (thick line), which is labeled by $\left(\mathbf{i}, \tau_{\mathbf{i}}=1\right)$. White, gray, and full circles represent in order $\hat{D}^{\dagger}, \hat{F}^{\dagger}$, and $\hat{P}^{\dagger}$ operators; the coefficients $\left(x_{l}, y_{l}, \epsilon\right.$, etc. $)$ are the numerical prefactors. 
This is simply obtained by taking the straight line $l_{\mathbf{y}+\mathbf{x}, v=1, n}$ crossing the site $\mathbf{i}$ and breaking the line at the site $\mathbf{i}$ in the breaking direction $\tau_{\mathbf{i}}=1$. Given by the periodic boundary conditions, a broken line besides its first cusp at $\mathbf{i}$, and $\tau_{\mathbf{i}}$ $=1$ (obtained by breaking the starting straight line at $\mathbf{i}$ ), will have a second cusp point at $\mathbf{j}=\mathbf{i}+(L / 2)(\mathbf{x}+\mathbf{y})$ and $\tau_{\mathbf{j}}=3$ (see Fig. 4), which is unambiguously determined by $\left(\mathbf{i}, \tau_{\mathbf{i}}\right)$. Hence, the obtained broken lines can be denoted by $l_{v}\left(\mathbf{i}, \tau_{\mathbf{i}}\right.$ $=1$ ) and their number for fixed $v$ becomes $L^{2} / 2$. Consequently, the broken line $l_{v}\left(\mathbf{i}, \tau_{\mathbf{i}}=1\right)$ is a continuous line in the sublattice $v$, which contains $L$ sites from $S_{v}$ and two cusps as presented in Fig. 4, where the cusp characterized by the breaking direction $\tau=1$ being present at the site $\mathbf{i}$.

\section{C. $W^{\dagger}$ operators}

The $\hat{W}_{v, \mathbf{i}, \tau, \lambda_{v}, e_{v}}^{\dagger}$ operator represents a linear combination of operators acting on $\mathbf{i}^{\prime}$ sites placed in an extended region surrounding the broken line $l_{v}\left(\mathbf{i}, \tau_{\mathbf{i}}=\tau\right)$, as presented in Figs. 5-7 for $\tau=1$. In Figs. 5-7, the black, open, and gray circles plotted at a site $\mathbf{i}^{\prime}$ are representing in order $\hat{P}_{\mathbf{i}^{\prime}, \lambda_{v}, e_{v}}^{\dagger}$ $=x_{\mathbf{i}^{\prime}}^{d} \hat{D}_{\mathbf{i}^{\prime}, \lambda_{v}, e_{v}}^{\dagger}+x_{\mathbf{i}^{\prime}}^{f} \hat{F}_{\mathbf{i}^{\prime}, \lambda_{v}, e_{v}}^{\dagger}, \hat{D}_{\mathbf{i}^{\prime}, \lambda_{v}, e_{v}}^{\dagger}$, and $\hat{F}_{\mathbf{i}^{\prime}, \lambda_{v}, e_{v}}^{\dagger}$ operators.

The coefficients present in Figs. 5-7 are numerical prefactors. In order to obtain the mathematical expression of the $\hat{W}_{v, \mathbf{i}, \tau=1, \lambda_{v}, e_{v}}^{\dagger}$ operator, one simply adds all contributions from Figs. 5-7 as follows:

$$
\begin{aligned}
\hat{W}_{v, \mathbf{i}, \tau=1, \lambda_{v}, e_{v}}^{\dagger}= & \xi \hat{F}_{\mathbf{i}-2 \mathbf{y}, \lambda_{v}, e_{v}}^{\dagger}+y_{0} \hat{D}_{\mathbf{i}-\mathbf{y}, \lambda_{v}, e_{v}}^{\dagger}+\epsilon \hat{D}_{\mathbf{i}+\mathbf{y}, \lambda_{v}, e_{v}}^{\dagger}+\mu \hat{F}_{\mathbf{i}+2 \mathbf{y}, \lambda_{v}, e_{v}}^{\dagger} \\
& +\sum_{n=0}^{M}\left(x_{n}^{d} \hat{D}_{\mathbf{i}+n(\mathbf{x}+\mathbf{y}), \lambda_{v}, e_{v}}^{\dagger}+x_{n}^{f} \hat{F}_{\mathbf{i}+n(\mathbf{x}+\mathbf{y}), \lambda_{v}, e_{v}}^{\dagger}\right) \\
& +\sum_{n=1}^{M-1}\left(x_{n}^{d} \hat{D}_{\mathbf{i}+n(\mathbf{y}-\mathbf{x}), \lambda_{v}, e_{v}}^{\dagger}+x_{n}^{f} \hat{F}_{\mathbf{i}+n(\mathbf{x}+\mathbf{y}), \lambda_{v}, e_{v}}^{\dagger}\right) \\
& +\sum_{n=1}^{M-1} y_{n} \hat{D}_{\mathbf{i}+n \mathbf{x}+(n-1) \mathbf{y}, \lambda_{v}, e_{v}}^{\dagger}+\sum_{n=1}^{M-1} y_{n} \hat{D}_{\mathbf{i}-n \mathbf{x}+(n-1) \mathbf{y}, \lambda_{v}, e_{v}}^{\dagger} \\
& +\sum_{n=2}^{M} y_{n} \hat{D}_{\mathbf{i}+(n-1) \mathbf{x}+n \mathbf{y}, \lambda_{v}, e_{v}}^{\dagger}+\sum_{n=2}^{M} y_{n} \hat{D}_{\mathbf{i}-(n-1) \mathbf{x}+n \mathbf{y}, \lambda_{v}, e_{v}}^{\dagger} \\
& +\gamma \hat{D}_{\mathbf{j}-\mathbf{y}, \lambda_{v}, e_{v}}^{\dagger}+\beta \hat{D}_{\mathbf{j}+\mathbf{y}, \lambda_{v}, e_{v}}^{\dagger}+\delta \hat{F}_{\mathbf{j}-2 \mathbf{y}, \lambda_{v}, e_{v}}^{\dagger} \\
& +\alpha_{1} \hat{F}_{\mathbf{j}+\mathbf{y}+\mathbf{x}, \lambda_{v}, e_{v}}^{\dagger}+\alpha_{1} \hat{F}_{\mathbf{j}+\mathbf{y}-\mathbf{x}, \lambda_{v}, e_{v}}^{\dagger}+\alpha_{0} \hat{F}_{\mathbf{j}+2 \mathbf{y}, \lambda_{v}, e_{v},}^{\dagger}
\end{aligned}
$$

where $M=L / 2, \mathbf{j}=\mathbf{i}+(L / 2)(\mathbf{x}+\mathbf{y})$, and effectuating the sums over the index $n$ in Eq. (16), the periodic boundary conditions must be taken into account. A similar procedure based on $\tau_{\mathbf{i}}=4, \tau_{\mathbf{j}}=2$ leads to the broken line used in constructing

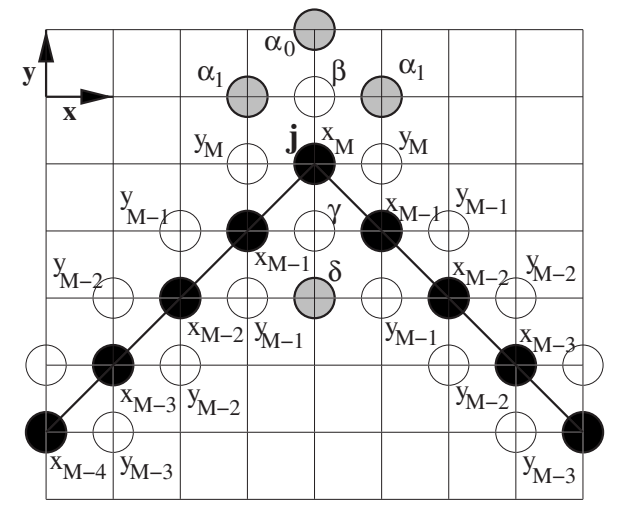

FIG. 6. The second cusp region of $\hat{W}_{v=1, \mathbf{i}, \tau=1, \lambda_{v}, e_{v}}^{\dagger}$. The thick line is a part of the broken line. For notations, see Fig. 5.
$\hat{W}_{v, \mathbf{i}, \tau=4, \lambda_{v}, e_{v}}^{\dagger}$, which can be obtained from $\hat{W}_{v, \mathbf{i}, \tau=1, \lambda_{v}, e_{v}}^{\dagger}$ by the interchange $\mathbf{x} \leftrightarrow \mathbf{y}$.

\section{Numerical prefactors of the $\hat{W}^{\dagger}$ operators}

The numerical prefactors present in Figs. 5-7 and Eqs. (16) are given as follows:

$$
\begin{gathered}
x_{M \geq n \geq 0}^{d}=(-1)^{n+1}(n+1) y_{0} \frac{a_{1, d}}{a_{2, d}}, y_{M \geq n \geq 1}=(-1)^{n} y_{0}, \\
x_{0}^{f}=\frac{y_{0}}{a_{1, f} a_{2, d}}\left(a_{1, d}^{2}+4 a_{2, d}^{2}\right), x_{1}^{f}=\frac{2 y_{0}}{a_{1, f} a_{2, d}}\left(a_{2, d}^{2}-a_{1, d}^{2}\right), \\
x_{M-2 \geq n \geq 2}^{f}=-\frac{a_{1, d}}{a_{1, f}} x_{n}^{d}, \epsilon=-3 y_{0}, \mu=-\xi=\frac{a_{2, d}}{a_{1, f}} y_{0}, \\
\alpha_{1}=-\frac{a_{2, d}}{a_{1, f}}\left(y_{M}-\frac{a_{2, d}}{a_{1, d}} x_{M}^{d}\right), \quad \beta=-\frac{a_{2, d}}{a_{1, d}} x_{M}^{d},
\end{gathered}
$$




$$
\begin{gathered}
\gamma=-\frac{a_{2, d}}{a_{1, d}}\left(2 x_{M-1}^{d}+x_{M}^{d}\right), \quad \delta=-\frac{a_{2, d}}{a_{1, f}}\left(\gamma+2 y_{M-1}\right), \\
x_{M-1}^{f}=-\frac{a_{1, d}}{a_{1, f}} x_{M-1}^{d}-\frac{a_{2, d}}{a_{1, f}}\left(2 y_{M-1}+y_{M}+\gamma\right), \\
x_{M}^{f}=-\frac{a_{1, d}}{a_{1, f}} x_{M}^{d}-\frac{a_{2, d}}{a_{1, f}}\left(2 y_{M}+\beta+\gamma\right), \alpha_{0}=\frac{a_{2, d}^{2} x_{M}^{d}}{a_{1, d} a_{1, f}},
\end{gathered}
$$

where $y_{0}$ is arbitrary and $x_{i}=\left(x_{i}^{d}, x_{i}^{f}\right)$.

The deduction procedure of Eq. (17) is as follows. (1) One fixes a $\hat{W}_{v, \mathbf{i}, 1, \lambda_{v}, e_{v}}^{\dagger}$ operator labeled by the fixed site $\mathbf{i}$ of the sublattice $v$, as shown by Figs. 5-7. (2) After this step, one takes all operators $\hat{A}_{\mathbf{i}^{\prime}, v^{\prime}, e^{\prime}}$ of the Hamiltonian from Eq. (7) which share common sites with $\hat{W}_{v, \mathbf{i}, 1, \lambda_{v}, e_{v}}^{\dagger}$, (3) calculate the anticommutators $\left\{\hat{A}_{\mathbf{i}^{\prime}, v^{\prime}, e^{\prime}}, \hat{W}_{v, \mathbf{i}, 1, \lambda_{v}, e_{v}}^{\dagger}\right\}$, and (4) require zero value for these, e.g.,

$$
\left\{\hat{A}_{\mathbf{i}^{\prime}, v^{\prime}, e^{\prime}}, \hat{W}_{v, \mathbf{i}, 1, \lambda_{v}, e_{v}}^{\dagger}\right\}=0,
$$

for all possible values of all indices. These conditions provide a linear system of equations, whose linearly independent terms build up the following system of equations.

In the region of the lower cusp, one has

$$
\begin{gathered}
a_{1, f} \xi+a_{2, d} y_{0}=0, \\
\epsilon a_{2, d}+a_{1, d} x_{0}^{d}+a_{1, f} x_{0}^{f}=a_{2, d} y_{0}, \\
a_{1, f} \mu+a_{2, d} \epsilon+2 y_{0} a_{2, d}=0, \\
a_{2, d}\left(y_{0}+\epsilon\right)+a_{1, d} x_{1}^{d}+a_{1, f} x_{1}^{f}=0, \\
a_{2, d} x_{0}^{d}+a_{1, d} y_{0}=0, \\
a_{1, d} x_{2}^{d}+a_{1, f} x_{2}^{f}=0, \\
a_{1, d} x_{3}^{d}+a_{1, f} x_{3}^{f}=0, \\
\epsilon a_{1, d}+a_{2, d}\left(x_{0}^{d}+2 x_{1}^{d}\right)=0, \\
a_{2, d}\left(x_{0}^{d}+x_{1}^{d}\right)+a_{1, d} y_{1}=0, \quad y_{1}=-y_{0}, \\
a_{2, d}\left(x_{1}^{d}+x_{2}^{d}\right)+a_{1, d} y_{2}=0, \quad y_{2}=y_{0}, \\
a_{2, d}\left(x_{2}^{d}+x_{3}^{d}\right)+a_{1, d} y_{3}=0, \quad y_{3}=-y_{0}, \\
a_{2, d}\left(x_{3}^{d}+x_{4}^{d}\right)+a_{1, d} y_{4}=0, \quad y_{4}=y_{0} .
\end{gathered}
$$

For the intercusp region presented in Fig. 7, one obtains for $4 \leq m \leq(M-2)$

$$
\begin{gathered}
a_{1, d} x_{m}^{d}+a_{1, f} x_{m}^{f}=0, \\
a_{2, d}\left(x_{m}^{d}+x_{m+1}^{d}\right)=-a_{1, d} y_{m+1}, \\
y_{m}=(-1)^{m} y_{0} .
\end{gathered}
$$

The upper cusp region provides the equations

$$
\begin{gathered}
a_{1, f} \delta+a_{2, d} \gamma=-2 a_{2, d} y_{M-1}, \\
2 a_{2, d} y_{M}+a_{2, d}(\beta+\gamma)+a_{1, d} x_{M}^{d}+a_{1, f} x_{M}^{f}=0, \\
a_{1, f} \alpha_{0}+a_{2, d} \beta=0, \\
a_{2, d}\left(\gamma+y_{M}\right)+a_{1, f} x_{M-1}^{f}=-2 a_{2, d} y_{M-1}-a_{1, d} x_{M-1}^{d}, \\
a_{2, d}\left(\beta+y_{M}\right)=-a_{1, f} \alpha_{1}, \\
a_{2, d}\left(y_{M}+y_{M-1}\right)=0, \\
a_{2, d} x_{M}^{d}+a_{1, d} \gamma=-2 a_{2, d} x_{M-1}^{d}, \\
a_{2, d} x_{M}^{d}+a_{1, d} \beta=0, \\
a_{2, d} x_{M}^{d}+a_{1, d} y_{M}=-a_{2, d} x_{M-1}^{d} .
\end{gathered}
$$

The system of equations [Eqs. (19)-(21)] must be solved considering $y_{0}$ a given but arbitrary quantity. The solution is presented in Eq. (17)

\section{E. Properties of the $W^{\dagger}$ operators}

The properties related to the constructed $W_{v, \mathbf{i}, \tau, \lambda_{v}, e_{v}}^{\dagger}$ operators are the following ones.

(a) Since $\left(L^{2} / 2\right)$ different possible sites $\mathbf{i} \in S_{v}$ are present in a given sublattice $v$, the operator $\hat{\phi}_{e_{v}}^{\lambda_{v}}(v)$ $=\Pi_{\mathbf{i} \in S_{v}}\left(\hat{W}_{v, \mathbf{i}, \tau=1, \lambda_{v}, e_{v}} \hat{W}_{v, \mathbf{i}, \tau=4, \lambda_{v}, e_{v}}^{\dagger}\right)$ introduces $L^{2}$ electrons into the system. Hence, based on $\left[\Pi_{v=1,2} \hat{\phi}_{e_{v}}^{\lambda_{v}}(v)\right]$, the vector from Eq. (15) becomes

$$
\left|\Psi_{e_{1}, e_{2}}^{\lambda_{1}, \lambda_{2}}\right\rangle=\left[\prod_{v=1,2} \hat{\phi}_{e_{v}}^{\lambda_{v}}(v)\right]|0\rangle
$$

and has $2 L^{2}$ electrons, e.g., which is defined at half-filling.

(b) The $2 L^{2}$ operators, $\hat{W}_{v, \mathbf{i}, \tau, \lambda_{v}, e_{v}}^{\dagger}$ present in Eq. (22) are constructed from $2 L^{2}$ canonical Fermi operators $\left(\hat{D}_{\mathbf{i}, \lambda_{v}, e_{v}}\right.$ and $\left.\hat{F}_{\mathbf{i}, \lambda_{v}, e_{v}}\right)^{25}$ and are linearly independent, since each of

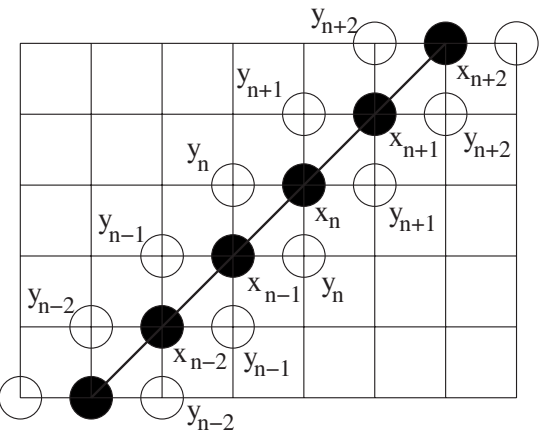

FIG. 7. Intercusps (straight) region of $\hat{W}_{v=1, \mathbf{i}, \tau=1, \lambda_{v}, e_{v}}^{\dagger}$ The thick line is a part of the broken line. For notations, see Fig. 5. 
them has at least one new segment ${ }^{26}$ in its construction.

(c) Since $W_{v, \mathbf{i}, \tau, \lambda_{v}, e_{v}}^{\dagger} W_{v, \mathbf{i}, \tau, \lambda_{v}, e_{v}}^{\dagger}=0$ holds, the operators $W_{v, \mathbf{i}, \tau, \lambda_{v}, e_{v}}$ are fermionic operators, but not satisfy canonical anticommutation rules, e.g., $\left\{W_{v, \mathbf{i}, \tau, \lambda_{v}, e_{v}}, W_{v^{\prime}, \mathbf{i}^{\prime}, \tau^{\prime}, \lambda_{v^{\prime}}^{\prime}, e_{v^{\prime}}^{\prime}}^{\dagger}\right\}$ $\neq \delta_{v, v^{\prime}} \delta_{\mathrm{i}, \mathrm{i}^{\prime}} \delta_{\tau, \tau^{\prime}} \delta_{\lambda, \lambda^{\prime}} \delta_{e, e^{\prime}}$.

(d) Given by the construction of $\hat{W}_{v, \mathbf{i}, \tau, \lambda_{v}, e_{v}}^{\dagger}$ operators [e.g., Eq. (18)], one has

$$
\hat{G}\left|\Psi_{e_{1}, e_{2}}^{\lambda_{1}, \lambda_{2}}\right\rangle=0
$$

hence, Eq. (15) gives the minimum energy eigenvalue (e.g., zero) of the first term of the studied Hamiltonian from Eq. (7).

(e) A quite important property of the $\hat{W}_{v, \mathbf{i}, \tau, \lambda_{v}, e_{v}}^{\dagger}$ operators is that they introduce $f$ electrons only in the sublattice $v$. This can be seen, for example, from Figs. 5-7 which best present the $\hat{W}^{\dagger}$ operators, by taking into account that $\hat{F}^{\dagger}$ operators (containing the $\hat{f}^{\dagger}$ operators) act only on sites depicted in Figs. 5-7 by gray and full (black) circles. Consequently, the $\hat{\phi}_{e_{v}}^{\lambda_{v}}(v)$ operator introduces $f$ electrons only in the sublattice $v$, and more than this, because of $\hat{F}_{\mathbf{i}, \lambda_{v}, e_{v}}^{\dagger} \hat{F}_{\mathbf{i}, \lambda_{v}, e_{v}}^{\dagger}$ $=0$, the vector $\hat{\phi}_{e_{v}}^{\lambda_{v}}(v)|0\rangle$ will contain only single $f$ occupancy in the sublattice $v$, while the other sublattice $v^{\prime} \neq v$ will contain only $d$ electrons. Concluding this point, the contributions created by $\hat{\phi}_{e_{v}}^{\lambda_{v}}(v)|0\rangle$ will have (i) in $S_{v}$ empty sites, single $d$ or $f$ occupancy, and double occupancy of the form $\hat{D}_{\mathbf{i}, \lambda_{v}, e_{v}}^{\dagger} \hat{F}_{\mathbf{i}, \lambda_{v}, e_{v}}^{\dagger}$ on sites $\mathbf{i} \in S_{v}$, while (ii) in the sublattice $S_{v^{\prime}}$, $v^{\prime} \neq v$ empty sites and single $d$ occupancy can appear.

(f) Because of the property mentioned at point (e), $\left|\Psi_{e_{1}, e_{2}}^{\lambda_{1}, \lambda_{2}}\right\rangle$ from Eq. (22) will contain $\hat{F}_{\mathbf{i}, \lambda_{1}, e_{1}}^{\dagger}$ operators in the sublattice $v=1, \mathbf{i} \in S_{1} ; \hat{F}_{\mathbf{j}, \lambda_{2}, e_{2}}^{\dagger}$ operators in the sublattice $v$ $=2, \mathbf{j} \in S_{2}$, hence double $f$ occupancy is not present, and consequently one has

$$
U \hat{U}_{f}\left|\Psi_{e_{1}, e_{2}}^{\lambda_{1}, \lambda_{2}}\right\rangle=0
$$

In conclusion, Eq. (15) gives the minimum energy eigenvalue (e.g., zero) also for the second term of the studied Hamiltonian from Eq. (7). Note that even if $\lambda_{1}, \lambda_{2}$ are arbitrary but fixed in Eq. (7) hence also in Eqs. (23) and (24), the parameters $e_{1}, e_{2}$ can be arbitrarily chosen.

(g) As seen in Figs. 4-7, the $\hat{W}^{\dagger}$ operators are extended. The extended nature has several aspects that have to be separately stressed. (1) First, it is not possible to find $2 L^{2}$ solutions of Eq. (18) for $\hat{W}^{\dagger}$ operators such to obtain $\hat{W}^{\dagger}$ enclosed in a cluster smaller than the size of the system and to preserve property (24). (2) By plotting all $\hat{W}^{\dagger}$ operators present for a fixed $L$, one realizes that for two arbitrary sites $\left(\mathbf{j}_{1}, \mathbf{j}_{2}\right)$ of the system, there is at least one $\hat{W}^{\dagger}$ operator containing both sites, hence representing a direct path between $\mathbf{j}_{1}$ and $\mathbf{j}_{2}$.

\section{GROUND STATE AT $U>0$}

\section{A. Ground state at half-filling}

The results presented in Sec. IV E [see Eqs. (23) and (24)] show that the ground state at half-filling of $\hat{H}$ is provided by the $\left|\Psi_{e_{1}, e_{2}}^{\lambda_{1}, \lambda_{2}}\right\rangle$ vectors. Since as specified in (f) of Sec. IV E, where $e_{1}, e_{2}= \pm 1$ can be arbitrarily taken, the ground state at half-filling becomes

$$
\left|\Psi_{g}\left(2 L^{2}\right)\right\rangle=\left|\Psi^{\lambda_{1}, \lambda_{2}}\right\rangle=\sum_{e_{1}, e_{2}= \pm 1}\left|\Psi_{e_{1}, e_{2}}^{\lambda_{1}, \lambda_{2}}\right\rangle,
$$

where the ground state energy being $E_{g}\left(2 L^{2}\right)=-2 L^{2} K_{d}$. Using Eqs. (9) and (10), one finds $K_{d}=\left(t_{2} / 2\right)\left[\left(V_{0} / V_{1}\right)^{2}+4\right]$, hence $E_{g}\left(2 L^{2}\right)=E_{g}^{0}\left(2 L^{2}\right)$ holds for arbitrary $U>0$. I stress here that since double $f$ occupancy is not present in the interacting ground state, only the presence of $U>0$ is required for $\left|\Psi_{g}\left(2 L^{2}\right)\right\rangle$, hence Eq. (25) is the ground state for arbitrary $U>0$.

Defining $\hat{\phi}^{\lambda_{v}}=\Sigma_{e_{v}} \hat{\phi}_{e_{v}}^{\lambda_{v}}$, the vector $\hat{\phi}^{\lambda_{v}}|0\rangle$ represents a singlet state for arbitrary $\lambda_{v}$. Since the ground state [Eq. (25)] becomes now $\left|\Psi_{g}\left(2 L^{2}\right)\right\rangle=\Pi_{v} \hat{\phi}^{\lambda_{v}}|0\rangle$, it represents a singlet, nonmagnetic state. Given by the arbitrary nature of the $\lambda_{1}, \lambda_{2}$ coefficients, one could write the ground state [Eq. (25)] also in the form $\left|\Psi_{g}\left(2 L^{2}\right)\right\rangle=\Sigma_{\lambda_{1}, \lambda_{2}} c_{\lambda_{1}, \lambda_{2}}\left|\Psi^{\lambda_{1}, \lambda_{2}}\right\rangle$, where $c_{\lambda_{1}, \lambda_{2}}$ are arbitrary numerical coefficients. However, this expression not emphasize a supplementary degeneracy given by the parameters $\lambda_{1}, \lambda_{2}$ only shows that the spin axis for both $v$ $=1,2$ can be arbitrarily chosen.

\section{B. One-particle localization length in the interacting case}

The obtained result in Eq. (25) shows that turning on an arbitrary $U>0$, the ground state at $\bar{n}=1 / 2$ has the property $d_{\mathbf{i}}^{f}=0$ on each site $\mathbf{i}$, where $d_{\mathbf{i}}^{f}=\left\langle\hat{n}_{\mathbf{i}, \uparrow}^{f} \hat{n}_{\mathbf{i}, \downarrow}^{f}\right\rangle$ and $\langle\ldots\rangle$ denotes ground state expectation value calculated in terms of $\left|\Psi_{g}\left(2 L^{2}\right)\right\rangle$. Consequently, since $\hat{d}_{\mathbf{i}}^{f}>0$ at $U=0$, the Hubbard repulsion, by strongly diminishing the $f$-electron double occupancy, spreads out the contributions in the ground state wave function (e.g., the ground state in exact terms can now be given only in terms of operators which extend along the whole system). The aspects of this fact have been underlined in point $(\mathrm{g})$ of of Sec. IV E. These properties are missing in the noninteracting case and are introduced by the Hubbard $U>0$. As a consequence, if one fixes two arbitrary sites $\left(\mathbf{j}_{n}, \mathbf{j}_{m}\right)$ of the system, there is at least one $\hat{W}_{v, \mathbf{i}, \tau, \lambda_{v}, e_{v}}^{\dagger}$ operator that contains both sites, hence represents a direct path between $\mathbf{j}_{n}$ and $\mathbf{j}_{m}$, e.g., gives

$$
\begin{aligned}
& \left\langle\hat{W}_{v, \mathbf{i}, \tau, \lambda_{v}, e_{v}}\left|\hat{b}_{\mathbf{j}_{n}, \sigma}^{\dagger} \hat{b}_{\mathbf{j}_{m}, \sigma}\right| \hat{W}_{v, \mathbf{i}, \tau, \lambda_{v}, e_{v}}\right\rangle\left\langle\left\langle\hat{W}_{v, \mathbf{i}, \tau, \lambda_{v}, e_{v}} \mid \hat{W}_{v, \mathbf{i}, \tau, \lambda_{v}, e_{v}}\right\rangle\right. \\
& \quad \sim O(1 / L),
\end{aligned}
$$

where $\left|\hat{W}_{v, \mathbf{i}, \tau, \lambda_{v}, e_{v}}\right\rangle=\hat{W}_{v, \mathbf{i}, \tau, \lambda_{v}, e_{v}}^{\dagger}|0\rangle$ and $r=\left|\mathbf{j}_{n}-\mathbf{j}_{m}\right|$ is arbitrary. ${ }^{27}$

This property produces a clear increase in the one-particle localization length $\ell$. I show this aspect by presenting an exemplification for $L=12$ in Fig. 8, where $\Gamma(\mathbf{r})$ from Eq. (1) calculated in terms of $\left|\Psi_{g}\left(2 L^{2}\right)\right\rangle$ is shown. This emphasizes 


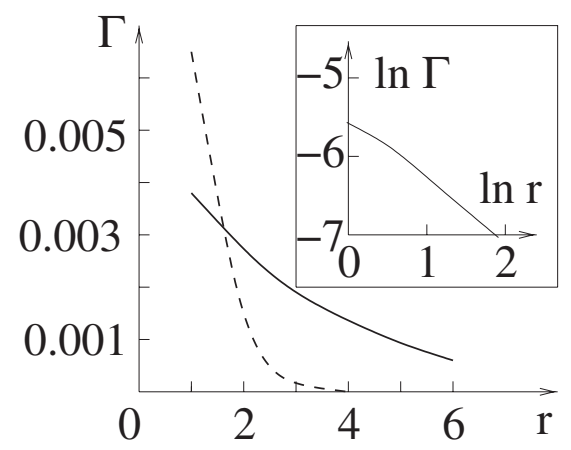

FIG. 8. Ground state expectation value of $f$-hopping correlations $\Gamma(\mathbf{r})$ at $U=0$ (dashed line) and $U \neq 0$ (continuous line) for a 12 $\times 12$ system at $t_{1} / t_{2}=10, t_{2} / V_{1}=0.5, V_{1} / V_{0}=0.1 . \mathbf{r} \|(\mathbf{x}+\mathbf{y})$ is in $\mid \mathbf{x}$ $+\mathbf{y} \mid$ units. The inset shows the $\log -\log$ plot at $U \neq 0$. For example, at $\mathbf{r}=4, \Gamma_{U} / \Gamma_{U=0}=2 \times 10^{5}$ holds (Ref. 28 ). The result is qualitatively the same for other directions and other $V_{1} / V_{0}$ ratios as well.

that $\Gamma(\mathbf{r})$ has no more strong exponential decay as in the $U$ $=0$ noninteracting case and can increase even 5 orders of magnitudes given by the presence of a nonzero $U$. The $\ell$ value corresponding to $\Gamma(\mathbf{r})$ for the interacting case in Fig. 8 (see inset) exceeds the sample size, while at $U=0$ had the value $\ell /|\mathbf{x}+\mathbf{y}| \sim 0.5$. The delocalization effect produced by the Hubbard $U>0$ is clearly seen.

The reason for this behavior is that $U$ throw the electrons in order to considerably diminish the double occupancy. This is why $\hat{W}^{\dagger}$ becomes extended along the whole system. In other words, it is not possible to construct at half-filling in the presence of $U>0 \hat{W}^{\dagger}$ operators with the properties presented in (e) of Sec. IV E, enclosed in a cluster with fixed extension smaller than the sample size. Concerning the needed degeneracy, I note the following: In order to construct the resulting extended $\hat{W}^{\dagger}$ operator from Eq. (16), the system must combine a huge number of linearly independent contributions. If all these contributions are belong to the same energy (e.g., the starting point is strongly degenerate), the creation of the interacting ground state containing the $\hat{W}^{\dagger}$ operators is without cost of energy, hence it is easier to be effectuated. This is the reason why macroscopic degeneracy is important for the process.

\section{Compensation effect in the periodic Anderson model}

I add at the end an observation regarding the compensation effect in the PAM which is also visible from the obtained solution. In the PAM, the $f$-electron moments are compensated in the singlet ground state at $\bar{n}=1 / 2$. Starting from the paper of Nozieres, ${ }^{29}$ the global or local nature of this compensation effect, especially in the large $U$ limit, is strongly debated. The presented results show that for arbitrary large repulsive $U$ values, the compensation can be manifestly global, being given by a subtle superposition effect of arbitrary spin orientations, involving both $b=d, f$ electrons.

\section{SUMMARY AND CONCLUSIONS}

In the study of the delocalization effect of the Hubbard interaction $U>0$, two aspects have to be analyzed in order to understand the physical reasons of this process: (i) how $U$ extends the one-particle states and (ii) how, given by $U$, the gap in the charge excitation spectrum disappears. For point (ii), the results deduced for the ionic Hubbard model at half-filling ${ }^{2-4}$ suggest that the correlation screening of the one-body potential responsible for the insulating phase can be invoked as an intuitive possibility for explanation. This aspect, however, not clearly emphasizes the physical reasons for point (i), namely, how the Hubbard repulsion extends the one-particle behavior in a genuine multielectronic and interacting case.

The presented paper provides physical insights regarding this aspect (i). First, one shows that always, when the delocalization effect of the Hubbard repulsion is observed, $U$ acts on the background of a macroscopic degeneracy in a multiband system. After this step one analyzes the delocalization effect produced on a band insulator starting point taken for the noninteracting case. For this, one considers a prototype two band model at half-filling, which at $U=0$ has a completely filled lowest band, separated by a gap from the empty upper band. In order to have the macroscopic degeneracy present, the lowest band has been chosen flat. After this step, $U$ has been turned on, and its effect analyzed. The extended nature of the one-particle behavior was tested by the ground state expectation value of the long-range hopping terms.

This analysis is made in the presented paper based on the periodic Anderson model (PAM) in two dimensions and halffilling as a prototype model. In order to provide valuable results, the study is presented at exact level. The PAM has been chosen since several aspects are known regarding the construction of its ground states, ${ }^{13,14}$ but, exact ground states for it in $2 \mathrm{D}$, interacting case, and half-filling, are deduced here. This is achieved by the use of extended operators, with the technique being described in the context of nonintegrable models.

The deduced results show that acting on the background of a macroscopic degeneracy, the Hubbard repulsion, by diminishing the double occupancy, spreads out the contributions in the ground state wave function. By this, it is able to considerably increase the one-particle localization length, hence the one-particle behavior becomes extended, which represents a clear physical source of the delocalization effect.

The study of point (ii) previously mentioned and related to the gap disappearance needs the deduction of the low laying excitation spectrum in the presented frame. The description of this case, at the level of the same quality, remains a challenging subject for future investigations.

\section{ACKNOWLEDGMENTS}

I am indebted to D. Vollhardt, A. P. Kampf, A. Zawadowski, and M. Gulacsi for support and discussions. Financial support has been provided by Alexander von Humboldt Foundation and Hungarian Scientific Research Fund under Contracts No. OTKA-T48782, 76821. 
${ }^{1}$ D. Vollhardt, in Perspectives in Many-Particle Physics, Proceedings of the CXXI Enrico Fermi International School, Varenna, 1992 (North-Holland, Amsterdam, 1994), p. 31.

${ }^{2}$ A. Garg, H. R. Krishnamurthy, and M. Randeria, Phys. Rev. Lett. 97, 046403 (2006).

${ }^{3}$ N. Paris, K. Bouadim, F. Hebert, G. G. Batrouni, and R. T. Scalettar, Phys. Rev. Lett. 98, 046403 (2007).

${ }^{4}$ S. S. Kancharla and E. Dagotto, Phys. Rev. Lett. 98, 016402 (2007).

${ }^{5}$ S. V. Kravchenko and M. P. Sarachik, Rep. Prog. Phys. 67, 1 (2004).

${ }^{6} \mathrm{~F}$. Gebhard, The Mott Metal-Insulator Transition, Models, and Methods, Springer Tracts in Modern Physics, Vol. 137 (Springer-Verlag, Berlin, 1997), p. 5.

${ }^{7}$ Z. Gulácsi, Phys. Rev. B 69, 054204 (2004).

${ }^{8}$ R. Resta and S. Sorella, Phys. Rev. Lett. 82, 370 (1999).

${ }^{9}$ M. Kollar, R. Strack, and D. Vollhardt, Phys. Rev. B 53, 9225 (1996).

${ }^{10}$ R. B. Laughlin, Phys. Rev. Lett. 50, 1395 (1983).

${ }^{11}$ Z. Gulácsi, A. Kampf, and D. Vollhardt, Phys. Rev. Lett. 99, 026404 (2007).

${ }^{12}$ R. Moessner and A. Ramirez, Phys. Today 59(2), 24 (2006).

${ }^{13}$ Z. Gulácsi and D. Vollhardt, Phys. Rev. Lett. 91, 186401 (2003).

${ }^{14}$ Z. Gulácsi and D. Vollhardt, Phys. Rev. B 72, 075130 (2005).

${ }^{15}$ Z. Gulácsi and M. Gulácsi, Phys. Rev. B 73, 014524 (2006).
${ }^{16}$ J. Cai and G. D. Mahan, Phys. Rev. B 76, 205116 (2007).

${ }^{17}$ Z. Gulácsi and I. Orlik, J. Phys. A 34, L359 (2001).

${ }^{18}$ P. Gurin and Z. Gulácsi, Phys. Rev. B 64, 045118 (2001).

${ }^{19}$ Z. Gulácsi, Phys. Rev. B 66, 165109 (2002).

${ }^{20}$ K. Held, C. Huscroft, R. T. Scalettar, and A. K. McMahan, Phys. Rev. Lett. 85, 373 (2000).

${ }^{21}$ C. Huscroft, A. K. McMahan, and R. T. Scalettar, Phys. Rev. Lett. 82, 2342 (1999).

${ }^{22}$ P. S. Riseborough, Phys. Rev. B 68, 235213 (2003).

${ }^{23}$ Z. Gulácsi, Eur. Phys. J. B 30, 295 (2002); I. Orlik and Z. Gulácsi, Philos. Mag. Lett. 78, 177 (1998).

${ }^{24}$ C. Sen, G. Alvarez, and E. Dagotto, Phys. Rev. B 70, 064428 (2004).

${ }^{25}$ The total number of these operators is $4 L^{2}$.

${ }^{26}$ Segment means here a linear combination of $\hat{D}^{\dagger}, \hat{F}^{\dagger}$ operators taken along a finite straight line segment.

${ }^{27}$ Note that $O(4 L)$ operators $\hat{B}^{\dagger}, B=D, F$ are present in a $\hat{W}^{\dagger}$ operator, and all coefficients in Eq. (17) have roughly the same order of magnitude fixed by $y_{0}$.

${ }^{28}$ For mathematical simplicity, $\lambda_{2}=-1 / \lambda_{1}^{*}$ (e.g., opposite spins in $v=1,2$ ) has been considered during the numerical calculation based on Eq. (25) of Fig. 8. Taking other $\left(\lambda_{1}, \lambda_{2}\right)$, the plot qualitatively remain the same.

${ }^{29}$ P. Nozieres, Ann. Phys. (Paris) 10, 19 (1985). 